\title{
Zinc Oxide Nanoparticle Suspensions and Layer-By- Layer Coatings Inhibit Staphylococcal Growth
}

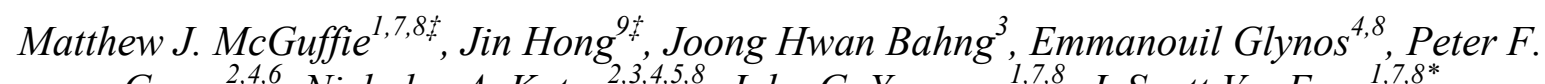
Green $^{2,4,6}$, Nicholas A. Kotov ${ }^{2,3,4,5,8}$, John G. Younger ${ }^{1,7,8}$, J. Scott VanEpps ${ }^{1,7,8^{*}}$

Departments of ${ }^{1}$ Emergency Medicine, ${ }^{2}$ Chemical Engineering, ${ }^{3}$ Biomedical Engineering, ${ }^{4}$ Materials Science and Engineering, ${ }^{5}$ Macromolecular Science and Engineering, and ${ }^{6}$ Applied Physics, ${ }^{7}$ Michigan Center for Integrative Research in Critical Care, and ${ }^{8}$ Biointerfaces Institute University of Michigan, 2800 Plymouth Rd, Ann Arbor, MI 48109, U.S.A.

${ }^{9}$ Key Laboratory of Biomedical Functional Materials, School of Sciences, China Pharmaceutical University, 639 Longmian Avenue, Nanjing 211198, China

$\$$ These authors contributed equally

*Corresponding Author:

University of Michigan,

Department of Emergency Medicine,

NCRC Building 26, Room327N,

2800 Plymouth Rd, Ann Arbor, MI 48109,

Phone (734) 763-2702

Fax (734) 763-9389

jvane@,med.umich.edu

Conflict of interest/funding: This work is partially supported by a Research Training Grant (RF2013-002) from the Society for Academic Emergency Medicine, National Institutes of Health Grant NIGMS RO1 GM081702, U.S. Army Research Office Grant Award No. W911NF10-1-0518, and AFOSR Grant Award No. MURI W911NF-12-1-0407. We acknowledge support from the National Science Foundation under grant ECS-0601345; CBET 0933384; CBET 0932823; and CBET 1036672. The authors have no competing conflicts of interest related to this work.

Abstract word count: 150

Manuscript word count: 4906

Number of references: 32

Number of figures: 8

Number of tables: 1

(C) 2015. This manuscript version is made available under the Elsevier user license http://www.elsevier.com/open-access/userlicense/1.0/ 


\begin{abstract}
:
Despite a decade of engineering and process improvements, bacterial infection remains the primary threat to implanted medical devices. Zinc oxide nanoparticles (ZnO-NPs) have demonstrated antimicrobial properties. Their microbial selectivity, stability, ease of production, and low cost make them attractive alternatives to silver NPs or antimicrobial peptides. Here we sought to (1) determine the relative efficacy of ZnO-NPs on planktonic growth of medically relevant pathogens; (2) establish the role of bacterial surface chemistry on ZnO-NP effectiveness; (3) evaluate NP shape as a factor in the dose-response; and (4) evaluate layer-bylayer (LBL) ZnO-NP surface coatings on biofilm growth. ZnO-NPs inhibited bacterial growth in a shape-dependent manner not previously seen or predicted. Pyramid shape particles were the most effective and contrary to previous work, larger particles were more effective than smaller particles. Differential susceptibility of pathogens may be related to their surface hydrophobicity. LBL coatings of $\mathrm{ZnO}-\mathrm{NP}$ reduced staphylococcal biofilm burden by $>95 \%$.
\end{abstract}

KEYWORDS: Zinc oxide, nanoparticles, layer-by-layer, biofilm, staphylococcus 


\section{BACKGROUND:}

The adhesion to and subsequent colonization of engineered materials by bacteria and bacterial biofilms pose significant challenges to many industries including, maritime shipping, naval engineering, waste water treatment, food safety, and healthcare. Biofilms are a particular threat to health where half of the 2 million annual healthcare-associated infections in the U.S. can be attributed to indwelling medical devices(1). Despite a decade of engineering advancements and clinical process improvements, infected devices remain the most common cause of healthcare-associated bloodstream infection(2). Treatment of implant infection requires surgical extraction of a potentially precious device and/or prolonged antibiotic therapy. Surgical replacement of a device brings significant risk for serious or even life-threatening complications. Extended courses of broad spectrum antibiotics bring other complications including toxicity (e.g., renal impairment) and opportunistic infections (e.g., Clostridium difficile colitis). Furthermore, extended antibiotic use drives the development of antibiotic resistance.

Data suggest that zinc oxide nanoparticles (ZnO-NPs) may have a role as anti-microbial agents(3-5) to prevent medical device infection(6, 7). Zinc oxide, in contrast to silver, is significantly less expensive. This is important since the use of rare materials in disposable medical devices can be cost prohibitive. In addition, the therapeutic window between efficacy and toxicity for silver is quite narrow. This has led to disappointing clinical effectiveness of silver-coated medical devices(8). ZnO-NPs on the other hand appear to have improved selectivity for bacteria over mammalian cells(9-11). In fact, $\mathrm{ZnO}$ is generally recognized as safe by the Federal Drug Administration. In comparison to antimicrobial peptides, which have also been evaluated extensively for this purpose, $\mathrm{ZnO}-\mathrm{NPs}$ are more stable, easier to prepare, and again significantly less expensive. This makes them a much more attractive alternative for 
device manufacturers who must consider the costs of regulatory approval and constraints of diminishing health care reimbursements. Indeed, a Cochrane review could not conclusively recommend widespread use of antibiotic impregnated catheters as the data seemed useful only in specific populations where the infection rate remains high despite the implementation of other less expensive interventions(12).

However, many questions regarding the use of $\mathrm{ZnO}-\mathrm{NPs}$ for this application remain. Specifically, what is the breadth of their antimicrobial spectrum? Given that nanoparticles must 'touch' the bacterial surface to work(13), how do microbial surface chemistry and nanoparticle shape contribute to $\mathrm{ZnO}-\mathrm{NP}$ antimicrobial function? Can $\mathrm{ZnO}-\mathrm{NPs}$ still provide anti-bacterial function when immobilized to a surface where they would likely increase the roughness (and potentially bacterial adhesiveness) of surfaces formed from standard device fabrication methods?

In this study we begin to address these significant hurdles to moving $\mathrm{ZnO}-\mathrm{NPs}$ forward as a new anti-infective coating for implanted medical devices alternative to silver and other lowmolecular weight antimicrobials. Specifically, we ask: (1) in bacterial suspension (a standard method for establishing antibiotic effectiveness), what is the relative efficacy against Staphylococcus aureus, Staphylococcus epidermidis, Escherichia coli, and Klebsiella pneumoniae? These organisms were chosen as they are the two most common Gram-positive and Gram-negative organisms, respectively, recovered from blood cultures in our emergency department annually(14). (2) How do bacterial surface properties (hydrophobicity and acid-base chemistry) relate to $\mathrm{ZnO}-\mathrm{NP}$ effectiveness? (3) As $\mathrm{ZnO}$ particles can be synthesized in various shapes, is there one shape that is most effective? As a corollary question, is there evidence that some other feature of the NP (e.g., shape, surface area) rather than simple mass concentration that may be the determining factor in the dose-response relationship? (4) When deposited on an 
in vitro model of a medical device surface, do $\mathrm{ZnO}-\mathrm{NPs}$ convey protection to bacterial contamination and biofilm development?

\section{METHODS}

Bacterial strains, media, and growth conditions: The bacterial strains used in this study were Escherichia coli UTI89 and MG1655, Klebsiella pneumoniae LM21, methicillin-resistant Staphylococcus aureus SH1000, and Staphylococcus epidermidis RP62A. Glycerol stocks of all strains maintained at $-80{ }^{\circ} \mathrm{C}$ were plated on tryptic soy agar, cultured overnight at $37^{\circ} \mathrm{C}$ and stored at $4{ }^{\circ} \mathrm{C}$. Single colony inoculates were grown in tryptic soy broth $+1 \%$ glucose $w / v$ (TSBG) under shaking conditions for 16 hours at $30^{\circ} \mathrm{C}$ and diluted 1:50 for planktonic growth curves and Calgary biofilm experiments.

ZnO-NP synthesis: ZnO-NPs were synthesized into three specific shapes, hexagonal pyramids, plates, and spheres (Figure 1). The various shapes were prepared using similar reactions without the use of surfactants or capping agents in order to minimize the effect of different surface chemistry and surface distribution of those molecules on the interaction with the bacterial cell surface. Specific reaction conditions are listed in Supplemental Table S1. Briefly, plates were synthesized by dissolving $5.5 \mathrm{~g} \mathrm{Zn}(\mathrm{Ac})_{2} \cdot 2 \mathrm{H}_{2} \mathrm{O}$ in $100 \mathrm{~mL}$ anhydrous methanol and heated to reflux for 1 hour. Then $1 \mathrm{~g} \mathrm{KOH}$ dissolved in $10 \mathrm{~mL}$ deionized water was added to the solution and then refluxed for 14 hours. Sphere synthesis was similar but the $\mathrm{KOH}$ was dissolved in anhydrous methanol instead of deionized water. Pyramids were synthesized as we have previously described(15) by first mixing $0.2 \mathrm{~g} \mathrm{KOH}$ with the $5.5 \mathrm{~g} \mathrm{Zn}(\mathrm{Ac})_{2} \cdot 2 \mathrm{H}_{2} \mathrm{O}$ before adding anhydrous methanol and refluxing for 48 hours. All NP precipitates were washed 3 times with anhydrous methanol and stored in the freeze-drier. 
Characterization of ZnO-NPs: ZnO-NP preparations were initially characterized by dynamic light scattering (DLS) using a Malvern Instruments Zetasizer Nano ZS to determine size distribution and zeta potential. However, the spherical NPs were quite small $(<4 \mathrm{~nm})$ which limited the accuracy of this method. Repeated DLS measurements of the spheres varied from 40nm-100nm. This overestimation compared to transmission electron microscopy (TEM) is likely a function of surrounding water shell and particle aggregation. Therefore, we abandoned further DLS measurements for the spheres. Detailed size measurements and selected area electron diffraction patterns of the ZnO-NPs were made using a JEOL 3011 Transmission Electron Microscope. TEM was not suitable for measuring the thickness of the plates. Attempts were made to use high resolution TEM with $30^{\circ}$ of $\alpha$-tilt as described in(16), however our microscope was only able to generate $20^{\circ}$ of tilt which has not sufficient to reliably determine the plate thickness. Therefore, the thickness of the $\mathrm{ZnO}$ plates was evaluated using a Bruker Dimension Icon atomic force microscope (AFM) with Scanasyst Air cantilever and Nanoscope Analysis 1.5 software. TEM and AFM samples were prepared by dropping the aqueous solution onto carbon TEM grids and drying at room temperature. NP surface chemistry was evaluated by photoluminescence (PL) and Fourier transform infrared spectroscopy (FTIR). PL spectra were obtained on a Jobin Yvon Horiba Fluoromax-3 instrument. For FTIR, powder samples of NPs were analyzed on a JASCO FT/IR-4100 instrument with attenuated total reflection crystals.

Bacterial ZnO-NP dose response growth curves: $\mathrm{ZnO}-\mathrm{NP}$ suspensions were prepared by sonicating $\mathrm{ZnO}-\mathrm{NPs}$ into TSBG for 30 minutes. The final $\mathrm{ZnO}-\mathrm{NP}$ concentrations were serial dilutions of a stock $\mathrm{ZnO}$ colloidal suspension. The maximum concentration used in this study $(667 \mu \mathrm{g} / \mathrm{ml})$ was the highest concentration that remained a stable colloidal suspension over the duration of the experiment under the specific experimental conditions. Bacterial growth was 
assessed by optical density at $600 \mathrm{~nm}\left(\mathrm{OD}_{600}\right)$ hourly for 10 hours in the presence of $\mathrm{ZnO}$-NPs. To summarize individual growth curves, a growth rate constant was calculated as the slope of the linear portion (i.e., exponential phase of growth) of the $\log _{2}\left(\mathrm{OD}_{600}\right)$ vs time data determined by linear regression. To evaluate growth inhibition versus bacterial killing in planktonic conditions, mid-log cultures of $S$. aureus were pelleted by centrifugation and resuspended in aqueous suspensions of ZnO-NPs. After 60 minute exposure the bacteria/ZnO-NP suspensions were serially diluted and plated on TSBG agar for colony enumeration.

Microbial Adhesion to Solvents (MATS) Assay: The MATS assay has been previously described in the literature(17). Bacteria were grown overnight in TSBG media, pelleted, and resuspended in PBS to $\mathrm{OD}_{600}$ of 0.6 for stationary phase. For mid-log phase the overnight culture was diluted 1:50 and grown for 4 hours prior to pelleting and resuspension at $\mathrm{OD}_{600}$ of 0.6. Bacterial cell suspensions $(1.2 \mathrm{ml})$ were vortex mixed for $90 \mathrm{~s}$ with various solvents $(0.2 \mathrm{ml})$. The mixture was allowed to stand for 15 min to ensure complete separation of the two phases before a sample was carefully removed from the aqueous phase and the $\mathrm{OD}_{600}$ measured. The percentage of bound cells was subsequently calculated by:

$$
\text { Partition Fraction }=\left(1-\frac{A}{A_{o}}\right) \times 100
$$

where $\mathrm{A}_{0}$ is the $\mathrm{OD}_{600}$ of the bacterial suspension before mixing and $\mathrm{A}$ is the $\mathrm{OD}_{600}$ after mixing.

To determine hydrophobicity the hydrophobic solvent hexadecane was used. The fraction of cells that partition to the hexadecane-aqueous interface is a measure of cell surface hydrophobicity. For the Lewis acid-base properties, a comparison between microbial cell migration to the solvent-aqueous interface for a monopolar (acidic or basic) solvent and an 
apolar solvent is made. Increased affinity for chloroform-aqueous interface over hexadecaneaqueous interface is a measure of cell surface electron donating properties (i.e., Lewis base). Increased affinity for diethyl ether-aqueous interface over a hexane-aqueous interface is a measure of cell surface electron accepting properties (i.e., Lewis base).

Bacterial zeta potential measurements: Early log-phase cultures of each bacterial strain were pelleted by centrifugation, washed twice, and suspended in phosphate buffered saline. Zeta potential measurements were made using a Malvern Instruments Zetasizer Nano ZS.

Layer-by-Layer (LBL) ZnO-NP surface coating: 96-well plate lids fit with polystyrene pegs (Calgary Biofilm Device) were coated with ZnO-NPs. Pegs were prepared using the UVO Cleaner (Jelight). NP suspensions were prepared by dissolving the appropriate NP in deionized water to a concentration of $0.1 \% \mathrm{w} / \mathrm{v}$. Polystyrene sulfonate (PSS) was dissolved in deionized water to a concentration of $5 \% \mathrm{w} / \mathrm{v}$. Prepared pegs were placed into NP suspensions for 30 minutes, rinsed with deionized water and quickly blown dry with nitrogen. Pegs were then placed in PSS solution for 5 minutes, rinsed and dried again. They were returned to NP suspension, and the process was repeated 10 times with the final coating being NPs.

Characterization of LBL ZnO-NP coatings: Adsorption of $\mathrm{ZnO}$ in each layer was confirmed by UV-vis spectroscopy using an 8453 UV-vis ChemStation Photospectrophotometer (Agilent Technologies). Completed LBL coatings were characterized by scanning electron microscopy (SEM) and AFM. For SEM, samples were fixed in gluteraldehyde, serially dehydrated in ethanol, air dried at room temp, sputter-coated with gold and visualized using AMRAY 1910 Field Emission Scanning Electron Microscope. For AFM, samples were imaged in tamping mode using the Asylum Research MFP-3D atomic force microscope. The NCH Pointprobe 
cantilevers by Nano World with a nominal spring constant and resonance frequency of $42 \mathrm{~N} / \mathrm{m}$ and $320 \mathrm{kHz}$, respectively were used. Roughness analysis of the AFM images was performed using the Asylum Research software. Goniometry measurements were taken using high resolution photographs of the contact angle between water and the LBL coated surfaces.

ZnO-NP Leaching from LBL surfaces: To confirm stability of the LBL coatings, polystyrene pegs with $\mathrm{ZnO}-\mathrm{NP}$ LBL coatings were incubated in either sterile water of PBS for a period of 7 days. $\mathrm{ZnO}$ leaching was quantified by absorbance at $350 \mathrm{~nm}\left(\mathrm{~A}_{350}\right)$ of the surrounding medium and compared to the $\mathrm{ZnO}-\mathrm{NP}$ suspension used for the coating process (positive control) and uncoated polystyrene pegs (negative control).

Conversion of mass concentration to surface area and particle number concentration: For each NP shape an idealized geometry was assumed based on Supplemental Figure S1 and the corresponding volumes and surface areas were calculated according to Supplemental Table S2. The particle number concentration was calculated as:

$$
\left[\text { Partice \#] }=\frac{[\text { Mass }]}{V \rho}\right.
$$

where $V$ is the particle volume and $\rho$ is the density of $\mathrm{ZnO}\left(5.6 \mathrm{~g} / \mathrm{cm}^{3}\right)$. The surface area concentration was calculated as:

$$
[\text { Surface Area }]=[\text { Particle \#]S }
$$

where $S_{A}$ is the particle surface area.

Bacterial surface colonization assay: Bacterial surface colonization was evaluated using the Calgary Biofilm Device as we have previously described(18-20). LBL ZnO-NP coated pegs 
were submerged in inoculated media for 16 hours at $37^{\circ} \mathrm{C}$. The pegs were removed, washed twice, and then sonicated for 10 minutes to liberate adherent bacteria. Quantitative culture was then performed to determine the colony forming units (CFUs) present on each peg. The limit of detection for this assay was 100 CFUs per square centimeter of peg. Pegs were also prepared for SEM as we have previously described(18).

Statistics: All data is presented as mean plus or minus standard error of the mean unless otherwise noted. For the MATS assay, experiments were performed in triplicate. Two-way ANOVA was performed with bacterial strain and growth phase as factors. For the Calgary biofilm experiments, one-way repeated measures ANOVA was performed for each bacterial strain to evaluate the effect of particle shape on bacteria recovered from the biofilms. In all cases, post-hoc pairwise testing was performed using the Tukey procedure with significance set at $\mathrm{p}<0.05$.

For the planktonic growth curve experiments, we performed linear mixed effects regression with log transformed optical density as the dependent variable, time as a fixed effect, and date of experiment as a random effect to calculate the growth rate constant. For comparison of dose response curves we again performed linear mixed effects regression with growth rate constant as the dependent variable, time and shape as fixed effects, and date of experiment as a random effect. Reported p-values represent the significance of shape as a predictor of the dose response.

\section{RESULTS:}

In this study, we considered three $\mathrm{ZnO}-\mathrm{NP}$ geometries: plates, spheres, and pyramids. The edges of the hexagonal base of pyramids were $\sim 20 \mathrm{~nm}$, while their side edges were $\sim 25 \mathrm{~nm}$ 
(Figure 1A). The diameter of spheres was $\sim 4.4 \mathrm{~nm}$ (Figure 1B). The diameter and thickness of plates were $\sim 20 \mathrm{~nm}$ and $\sim 3.5 \mathrm{~nm}$, respectively (Figure 1C \& Supplemental Figure S2). Despite the obvious differences in the shape of the NPs, the crystals structures were nearly identical and all diffraction rings could be matched to the hexagonal phase of bulk $\mathrm{ZnO}$ (JCPDS 36-1451)(21) (Figure 1D-F). In addition, there were no marked chemical differences seen in PL and FTIR spectra for the three NP shapes (Figure 2).

Planktonic growth curves were generated for each bacterial strain in the presence of escalating mass concentrations of each ZnO-NP shape (Figure 3). The Gram-positive organisms (i.e., S. aureus and S. epidermidis) showed a dose-dependent reduction in growth for all three NP shapes. The Gram-negative organisms (i.e., E. coli and K. pneumonia) were not affected by the presence of $\mathrm{ZnO}-\mathrm{NPs}$ up to $667 \mu \mathrm{g} / \mathrm{ml}$. Enumeration of CFUs after 60 minute exposure to $\mathrm{ZnO}$ NP suspensions demonstrated 1-2 $\log _{10}$ reduction in CFU/ml from control for all three shapes at $667 \mu \mathrm{g} / \mathrm{ml}$ (Supplemental Figure S3). For pyramids and plates there was a saturation effect at $500 \mu \mathrm{g} / \mathrm{ml}$, where no additional killing was observed at the higher dose.

To examine the role of bacterial surface chemistry on $\mathrm{ZnO}-\mathrm{NP}$ antibacterial function, we used the well-established microbial adhesion to solvents assay (MATS) to determine the surface hydrophobicity (Figure 4) and Lewis acid-base properties (Supplemental Figure S4) of the four test organisms. The two Gram-positive species tested were found to be highly hydrophobic, with near complete migration $(99 \% \pm 1 \%$ for $S$. epidermidis and $98 \% \pm 2 \%$ for $S$. aureus) to the aqueous-hexadecane interface. The opposite was observed with E. coli and $K$. pneumoniae, for which no more than $10 \%(6.6 \% \pm 7 \%$ and $0 \%$ respectively) of suspended organisms were found at the solvent interface after extensive mixing. There were no significant differences in hydrophobicity measured during mid-log versus stationary phase of growth. 
The Gram-positive organisms had extreme hydrophobicity and therefore minimal Lewis acid-base interactions (Supplemental Figure S4A). E. coli and K. pneumonia had similar partitioning to the chloroform-aqueous interface during mid-log growth indicating a modest electron donating capacity on their surface $(29 \% \pm 2 \%$ and $24 \% \pm 7 \%$ respectively). During stationary phase, E. coli had increased migration to the chloroform-aqueous interface $(71 \% \pm$ $7 \%)$ while $K$. pneumonia had a slight but insignificant decrease $(20 \% \pm 2 \%)$ when compared to mid-log phase (Supplemental Figure S4B). E. coli and K. pneumonia also had similar migration to the diethyl ether-aqueous interface at midlog $(33 \% \pm 9 \%$ and $30 \% \pm 2 \%$ respectively) and stationary phase $(28 \% \pm 1 \%$ and $28 \% \pm 7 \%$ respectively) indicating modest electron accepting capacity Supplemental (Figure S4C).

To summarize the growth curve data in Figure 3 and make direct comparisons of the three NP shapes we calculated a growth rate constant for each dose of each NP shape. To determine if some other feature of the NP (e.g., shape, surface area) may be the determining factor in the dose-response we converted the mass concentrations used for the planktonic growth curves to surface area and molar concentrations based on the TEM measurements (Figure 1) and known density of $\mathrm{ZnO}$. While the same mass of pyramid, plate, and sphere NPs was used in each experiment, those masses converted to large differences in available surface area and total particle number (Table 1). As a quantitative measure of the antibacterial effect of a certain dose and specific shape, we plotted the growth rate constant against the mass, surface area, and particle concentrations for each of the three NP shapes (Figure 5). The effectiveness of a given dose was determined by the reduction in growth rate constant. For mass concentration, the dose response for spheres and pyramids were essentially equal $(\mathrm{p}=0.96)$ while plates had a somewhat attenuated effectiveness $(\mathrm{p}<0.05$, Figure 5A). However, for surface area and particle number 
concentration, pyramids had the greatest dose response followed by plates and then spheres $\left(\mathrm{p}<10^{-3}\right.$, Figure $\left.5 \mathrm{~B} \& \mathrm{C}\right)$.

The application of ZnO-NPs to surfaces using the LBL technique was confirmed by UVvis spectropscopy (example data shown in Supplemental Figure S5). The increase in absorption at $350 \mathrm{~nm}$ per layer varied between $0.04-0.05$ for plates and spheres and $0.02-0.03$ for spheres. This difference is thought to be related the fact that the smaller spheres create thinner $\mathrm{ZnO}$ layers and therefore less absorption. Final LBL surface coatings of ZnO-NPs were characterized by SEM, AFM, and goniometry. All ZnO-NP shapes increased roughness over the starting substrate (Supplemental Figure S6, S7, and Table S3). Contact angles varied from $18^{\circ}$ for plates to $56^{\circ}$ for pyramids (Table S3). These coatings remained stable with no measureable leaching of $\mathrm{ZnO}$ into PBS or water over 7 days (Figure 6).

Despite the increased surface roughness, we demonstrated a dramatic reduction in bacterial burden on polystyrene coated with ZnO-NPs. The coated surfaces had $>95 \%\left(\mathrm{p}<10^{-3}\right)$ reduction in the number $S$. aureus and $S$. epidermidis but not $E$. coli cells recovered when compared to bare surfaces (Figure 7). There were no significant NP shape effects on biofilm inhibition of S. aureus. On the other hand, spheres had a significantly greater inhibitory effect over plates for S. epidermidis ( $99.5 \%$ vs $98.5 \%$, p 0.005).

To better understand how the bacteria are interacting with the surfaces, we performed SEM on surfaces after biofilm culture (Figure 8). These images demonstrate bacterial adhesion and biofilm development on bare surfaces (Figure 8A-C) particularly for S. epidermidis (Figure 8C). However, despite an almost 3 log reduction in the number of viable cells recovered from the $\mathrm{ZnO}-\mathrm{NP}$ coated surfaces (Figure 7) there continues to be intact cells visualized on SEM 
(Figure 8D-L). In particular, there was a $99.5 \%$ reduction in S. epidermidis recovered from the $\mathrm{ZnO}$ sphere coated surface but persistence of biofilm appearance on SEM (Figure 9F).

\section{DISCUSSION:}

ZnO-NPs are a potential new antimicrobial technology with many features which make them an attractive alternative to silver or antimicrobial peptides for preventing medical device infection. In this study, we synthesized ZnO-NPs into three distinct shapes without the use of traditional surfactants or capping agents. This feature of our synthesis process is significant in light of the potential for these additional molecules to confound the results of experiments. As such, we were able to synthesize $\mathrm{ZnO}$-NPs with high crystallinity and nearly identical surface chemistry, differing only in shape and size.

The current literature suggests that $\mathrm{ZnO}-\mathrm{NPs}$ have a broad antimicrobial spectrum of action which include Gram-positive, Gram-negative, and spore forming organisms(3-5). Therefore, we hypothesized that all four test organisms would be susceptible to the $\mathrm{ZnO}-\mathrm{NPs}$. Our $\mathrm{ZnO}-\mathrm{NP}$ suspensions selectively inhibit the growth of Gram-positive organisms including methicillin resistant S. aureus (MRSA). While others have also shown a dose-dependent selectivity of ZnO-NPs for Gram-positive organisms(9), there are multiple studies demonstrating growth inhibition of Gram-negative organisms including E. coli(22-24). We attribute this discrepancy to three possible phenomena. The first is the use of surfactants and capping agents for NP synthesis which likely change the surface energies of both bacteria and NPs and therefore modulate the free energy of interaction. Indeed, the $\mathrm{ZnO}$-NP synthesis media used by Brayner et al. led to membrane disruption in the absence of nanoparticles(25). Second, in many cases, previously tested strains of $E$. coli are laboratory strains or expression vectors that lack the 
clinically ubiquitous capsule or surface proteins which may provide protection against the $\mathrm{ZnO}$ $\operatorname{NPs}(3,26)$. Finally, the molar dose of ZnO-NPs used in previous studies $(4,5,11,27)$ was much larger $(1-6 \mathrm{mM})$ than that used here $(23 \mathrm{nM}-6 \mu \mathrm{M})$. Gram-negative organisms may require a higher particle number for bacterial inhibition. Reddy et al, showed that E. coli required $>3.4 \mathrm{mM} \mathrm{ZnO}$-NPs for complete inhibition whereas $S$. aureus only required $1 \mathrm{mM}(11)$. However, these higher concentrations may be toxic to mammalian cells. For instance, human T-cells begin to show toxicity to $\mathrm{ZnO}-\mathrm{NPs}$ at concentrations around $5 \mathrm{mM}(10)$. The effective concentrations used here were at least 1000-fold lower.

The current consensus is that $\mathrm{ZnO}-\mathrm{NPs}$ work through contact with the bacterial surface which leads to either cell membrane disruption or generation of reactive oxygen species(13). Assuming this mechanism to be true, the surface chemistry of a bacterium will likely influence the potential for interaction between the NP and the bacterium. To that end, we examined how bacterial surface chemistry may contribute to ZnO-NP susceptibility. The striking contrast in surface hydrophobicity between Gram-positive and Gram-negative organisms parallels their susceptibility to ZnO-NPs. Furthermore, it provides evidence for a mechanism by which surfactants or capping molecules alter surface interactions and thereby antibacterial function of ZnO-NPs. Of note, we did observe the presence of both electron donating and accepting properties in the Gram-negative organisms. This is likely a result of the heterogeneous composition of the cell surface and the presence of zwitterions. Therefore, the acid-base surface chemistry of a cell is a function of the $\mathrm{pH}$ of the surrounding media. All experiments in this case were performed with normal PBS at $\mathrm{pH}$ 7.4. These results are complementary to work by Arakha et al., who demonstrated that ZnO-NP antimicrobial efficacy depended on interfacial interaction between the nanoparticle and the bacterium(28). Specifically, ZnO-NPs with positive 
zeta potentials had antimicrobial effects while negatively charged NPs did not. The positively charge NPs are able to interact with the negatively charged bacteria. The zeta potentials for the NPs in this study were positive in all cases (Supplementary Table S1). Zeta potentials for most bacteria, including those studied here, at physiologic $\mathrm{pH}$ are negative (Supplementary Table S4). This ensures a high likelihood of interaction between bacteria and NP for all strains tested. However, given the relative high salt concentration of culture media and the resulting short Debye lengths, the subtle differences in zeta potential between different bacterial strains are unlikely to have the profound differential observed between Gram-positive and Gram-negative bacteria.

It has previously been shown that decreasing particle size (and increasing affective surface area) leads to increase antimicrobial efficacy(4) while differences in particle shape may alter function to a much lesser degree $(27,29)$. However, it is difficult to sort out the relative contributions of size and shape. This is further confounded by the use of different capping agents to modify particle shape. Stankovic et al. showed that the antibacterial activity of $\mathrm{ZnO}-$ NPs depends on their synthesis method and the resulting morphology and surface area(27). However, they also used a variety of stabilizing agents which may have contributed to the observed differences. Our ability to synthesize three different NP shapes with similar crystalline structure and surface chemistry allowed us to better isolate the role of shape in the antibacterial properties of $\mathrm{ZnO}-\mathrm{NPs}$.

In general, when shape and size have been considered, the smallest NP, regardless of shape, appears to be the most effective (27, 30). Therefore, we hypothesized that the $\mathrm{ZnO-NP}$ spheres which are small and have high surface area to weight ratio would be the most effective. However in this study, $\mathrm{ZnO}$ pyramids at the same mass concentration as other shaped particles 
had greater or equal effectiveness with less surface area and less particle number. Our data suggest that shape may play a more important role than previously considered. In light of the previous discussion regarding the modulating effects of stabilizing molecules, previous work comparing different shapes may have been confounded by the synthesis technique. Since the NPs synthesized here were devoid of surfactant or stabilizing molecules (with the exception of $\mathrm{KOH}$ which was used in all three preparations) a more direct comparison of shape was possible. Indeed, the most effective NP shape on a molar concentration basis was not the smallest (spheres), rather the largest (pyramids). This work highlights the difficulty in teasing out the independent contribution of size and shape on $\mathrm{ZnO}-\mathrm{NP}$ antibacterial efficacy and the need to carefully consider the appropriate units of dose in studies of nanoparticles as medical therapeutics.

Given the capacity of $\mathrm{ZnO}-\mathrm{NPs}$ to inhibit planktonic bacterial growth we sought to translate this technology to surfaced based biofilm inhibition. We employed a LBL technique to immobilize the ZnO-NPs to a polymer substrate. This is the first use of LBL ZnO-NPs as an antibacterial coating. To our knowledge only one other group has immobilized ZnO-NPs to a surface with intent to prevent biofilm formation $(6,7)$. Their technique was limited to a glass substrate and required high energy ultrasound and cavitation. Our technique is simple, inexpensive, and can be applied to many different polymer surfaces used for medical devices. The coating was shown to be stable with no leaching of $\mathrm{ZnO}$ to the surrounding solution over one week. While we specifically did not evaluate for dissolution to $\mathrm{Zn}$ ions, it has been previously determined that high concentrations of $\mathrm{Zn}$ ions are not bacteriocidal(26). Therefore, dissolved $\mathrm{Zn}$ ions in the surrounding solution would not likely be contributing to the antimicrobial effects. 
A particular concern for this application is that NP coatings will likely increase surface roughness and therefore bacterial adhesion(31). Indeed, all our ZnO-NP coated surfaces were significantly rougher than the bare substrate. However, we had dramatic reductions (>95\%) in the numbers of viable bacteria recovered from $\mathrm{ZnO}-\mathrm{NP}$ coatings. This is comparable to the antibacterial performance of chlorhexidine-silver sulfadiazine coated catheters currently used clinically(32). SEM was used to better visualize the interactions of cells with the surfaces. However, it should be noted that SEM is limited in that it cannot differentiate living from dying cells. Given this limitation, we see cells of unclear viability adhering to the surfaces coated with ZnO-NPs. However, based on the quantitative culture, the cells dispersed from the $\mathrm{ZnO}-\mathrm{NP}$ coated surfaces are no longer viable (i.e., able to form a colony). That is, the $\mathrm{ZnO}$ coated surfaces may promote adhesion but lead to contact killing. Since the ZnO-NPs are not leaching from the surface and $\mathrm{Zn}$ ions are not especially toxic, the growth inhibition would have to occur only at the surface. To be sure, SEM images before and after dispersion for quantitative culture demonstrated that the majority of cells were indeed removed from all surfaces and that our quantitative culture results are not biased by the ability to disperse the cells from the surface. Of note, it is possible for the cells to have a viable but uncultureable phenotype which could not be differentiated by this analysis.

In conclusion, ZnO-NPs reduce planktonic growth of Gram-positive but not Gramnegative bacteria in a dose-dependent manner. This differential effect may be related to bacterial surface hydrophobicity. Shape modulates the dose response for ZnO-NPs when particle number or surface area are used as dosing units. LBL coating of polystyrene with ZnO-NP reduces staphylococcal biofilm burden despite increased in surface roughness and likely bacterial adhesion. This work furthers $\mathrm{ZnO}$-NPs as alternative medical device coating materials. 


\section{ACKNOWLEDGMENTS:}

The authors would like to thank Gleiciani de Queiros Silverira for her help with identification of electron diffraction rings for crystalline $\mathrm{ZnO}$, Usha Kadiyala for her assistance completing the quantitative culture of S. aureus, and Siu On Tung for his assistance with obtaining FTIR spectra. 


\section{REFERENCES}

1. Darouiche RO. Treatment of Infections Associated with Surgical Implants. New England Journal of Medicine. 2004;350(14):1422-9. PubMed PMID: 15070792.

2. Maki DG, Kluger DM, Crnich CJ. The Risk of Bloodstream Infection in Adults With Different Intravascular Devices: A Systematic Review of 200 Published Prospective Studies. Mayo Clinic Proceedings. 2006 9//;81(9):1159-71.

3. Zhang L, Jiang Y, Ding Y, Povey M, York D. Investigation into the antibacterial behaviour of suspensions of $\mathrm{ZnO}$ nanoparticles ( $\mathrm{ZnO}$ nanofluids). J Nanopart Res. 2007 2007/06/01;9(3):479-89. English.

4. Raghupathi KR, Koodali RT, Manna AC. Size-dependent bacterial growth inhibition and mechanism of antibacterial activity of zinc oxide nanoparticles. Langmuir : the ACS journal of surfaces and colloids. 2011 Apr 5;27(7):4020-8. PubMed PMID: 21401066. Epub 2011/03/16. eng.

5. Jones N, Ray B, Ranjit KT, Manna AC. Antibacterial activity of ZnO nanoparticle suspensions on a broad spectrum of microorganisms. FEMS Microbiology Letters. 2008;279(1):71-6.

6. Applerot G, Perkas N, Amirian G, Girshevitz O, Gedanken A. Coating of glass with $\mathrm{ZnO}$ via ultrasonic irradiation and a study of its antibacterial properties. Applied Surface Science. 2009 11/15/;256(3, Supplement):S3-S8.

7. Applerot G, Lellouche J, Perkas N, Nitzan Y, Gedanken A, Banin E. ZnO nanoparticlecoated surfaces inhibit bacterial biofilm formation and increase antibiotic susceptibility. RSC Advances. 2012;2(6):2314-21. 
8. Politano AD, Campbell KT, Rosenberger LH, Sawyer RG. Use of silver in the prevention and treatment of infections: silver review. Surgical infections. 2013 Feb;14(1):8-20. PubMed PMID: 23448590. Epub 2013/03/02. eng.

9. Premanathan M, Karthikeyan K, Jeyasubramanian K, Manivannan G. Selective toxicity of $\mathrm{ZnO}$ nanoparticles toward Gram-positive bacteria and cancer cells by apoptosis through lipid peroxidation. Nanomedicine : nanotechnology, biology, and medicine. 2011 Apr;7(2):184-92. PubMed PMID: 21034861.

10. Hanley C, Layne J, Punnoose A, Reddy KM, Coombs I, Coombs A, et al. Preferential killing of cancer cells and activated human $\mathrm{T}$ cells using $\mathrm{ZnO}$ nanoparticles. Nanotechnology. 2008;19(29):295103. PubMed PMID: PMC2558672.

11. Reddy KM, Feris K, Bell J, Wingett DG, Hanley C, Punnoose A. Selective toxicity of zinc oxide nanoparticles to prokaryotic and eukaryotic systems. Applied Physics Letters. 2007;90(21):-

12. Lai NM, Chaiyakunapruk N, Lai NA, O'Riordan E, Pau WS, Saint S. Catheter impregnation, coating or bonding for reducing central venous catheter-related infections in adults. The Cochrane database of systematic reviews. 2013;6:CD007878. PubMed PMID: 23740696. Epub 2013/06/07. eng.

13. Neal AL. What can be inferred from bacterium-nanoparticle interactions about the potential consequences of environmental exposure to nanoparticles? Ecotoxicology (London, England). 2008 Jul;17(5):362-71. PubMed PMID: 18454313. Epub 2008/05/06. eng.

14. Halverson S, Malani PN, Newton DW, Habicht A, Vander Have K, Younger JG. Impact of hourly emergency department patient volume on blood culture contamination and 
diagnostic yield. Journal of clinical microbiology. 2013 Jun;51(6):1721-6. PubMed PMID: 23515549. Pubmed Central PMCID: PMC3716046. Epub 2013/03/22. eng.

15. Yang M, Sun K, Kotov NA. Formation and Assembly-Disassembly Processes of $\mathrm{ZnO}$ Hexagonal Pyramids Driven by Dipolar and Excluded Volume Interactions. Journal of the American Chemical Society. 2010 2010/02/17;132(6):1860-72.

16. Palchoudhury S, Xu Y, Rushdi A, Holler RA, Bao Y. Controlled synthesis of iron oxide nanoplates and nanoflowers. Chemical Communications. 2012;48(85):10499-501.

17. Bellon-Fontaine MN, Rault J, van Oss CJ. Microbial adhesion to solvents: a novel method to determine the electron-donor/electron-acceptor or Lewis acid-base properties of microbial cells. Colloids and Surfaces B: Biointerfaces. 1996 7/31/;7(1-2):47-53.

18. Satorius AE, Szafranski J, Pyne D, Ganesan M, Solomon MJ, Newton DW, et al. Complement c5a generation by staphylococcal biofilms. Shock (Augusta, Ga). 2013 Apr;39(4):336-42. PubMed PMID: 23459111. Epub 2013/03/06. eng.

19. Orizondo RA, Babcock CI, Fabiilli ML, Pavlovsky L, Fowlkes JB, Younger JG, et al. Characterization of a Reverse-Phase Perfluorocarbon Emulsion for the Pulmonary Delivery of Tobramycin. Journal of aerosol medicine and pulmonary drug delivery. 2014 Jan 29. PubMed PMID: 24476046. Epub 2014/01/31. Eng.

20. Ceri H, Olson ME, Stremick C, Read RR, Morck D, Buret A. The Calgary Biofilm Device: new technology for rapid determination of antibiotic susceptibilities of bacterial biofilms. Journal of clinical microbiology. 1999 Jun;37(6):1771-6. PubMed PMID: 10325322. Pubmed Central PMCID: PMC84946. Epub 1999/05/15. eng. 
21. Moussodia R-O, Balan L, Merlin C, Mustin C, Schneider R. Biocompatible and stable ZnO quantum dots generated by functionalization with siloxane-core PAMAM dendrons. Journal of Materials Chemistry. 2010;20(6):1147-55.

22. Sawai J, Igarashi H, Hashimoto A, Kokugan T, Shimizu M. Evaluation of Growth Inhibitory Effect of Ceramics Powder Slurry on Bacteria by Conductance Method. Journal of Chemical Engineering of Japan. 1995;28(3):288-93.

23. Sawai J, Igarashi H, Hashimoto A, Kokugan T, Shimizu M. Effect of Particle Size and Heating Temperature of Ceramic Powders on Antibacterial Activity of Their Slurries. Journal of Chemical Engineering of Japan. 1996;29(2):251-6.

24. Sawai J, Saito I, Kanou F, Igarashi H, Hashimoto A, Kokugan T, et al. Mutagenicity Test of Ceramic Powder Which Have Growth Inhibitorory Effect on Bacteria. Journal of Chemical Engineering of Japan. 1995;28(3):352-4.

25. Brayner R, Ferrari-Iliou R, Brivois N, Djediat S, Benedetti MF, Fiévet F. Toxicological Impact Studies Based on Escherichia coli Bacteria in Ultrafine ZnO Nanoparticles Colloidal Medium. Nano Letters. 2006 2006/04/01;6(4):866-70.

26. Zhang L, Jiang Y, Ding Y, Daskalakis N, Jeuken L, Povey M, et al. Mechanistic investigation into antibacterial behaviour of suspensions of $\mathrm{ZnO}$ nanoparticles against $\mathrm{E}$. coli. J Nanopart Res. 2010 2010/06/01;12(5):1625-36. English.

27. Stankovic A, Dimitrijevic S, Uskokovic D. Influence of size scale and morphology on antibacterial properties of $\mathrm{ZnO}$ powders hydrothemally synthesized using different surface stabilizing agents. Colloids and surfaces B, Biointerfaces. 2013 Feb 1;102:21-8. PubMed PMID: 23010107. Epub 2012/09/27. eng. 
28. Arakha M, Saleem M, Mallick BC, Jha S. The effects of interfacial potential on antimicrobial propensity of ZnO nanoparticle. Sci Rep. 2015 04/15/online;5.

29. Zhang L, Ding Y, Povey M, York D. ZnO nanofluids - A potential antibacterial agent. Progress in Natural Science. 2008 8/10/;18(8):939-44.

30. Ramani M, Ponnusamy S, Muthamizhchelvan C, Cullen J, Krishnamurthy S, Marsili E. Morphology-directed synthesis of $\mathrm{ZnO}$ nanostructures and their antibacterial activity. Colloids and surfaces B, Biointerfaces. 2013 May 1;105:24-30. PubMed PMID: 23352944. Epub 2013/01/29. eng.

31. Singh AV, Vyas V, Patil R, Sharma V, Scopelliti PE, Bongiorno G, et al. Quantitative Characterization of the Influence of the Nanoscale Morphology of Nanostructured Surfaces on Bacterial Adhesion and Biofilm Formation. PLoS ONE. 2011;6(9):e25029.

32. Darouiche RO, Mansouri MD, Gawande PV, Madhyastha S. Antimicrobial and antibiofilm efficacy of triclosan and DispersinB combination. The Journal of antimicrobial chemotherapy. 2009 Jul;64(1):88-93. PubMed PMID: 19447791. 


\section{FIGURE LEGENDS:}

Figure 1. Representative (A-C) TEM images and (D-F) selected area electron diffraction patterns of $\mathrm{ZnO}(\mathrm{A} \& \mathrm{D})$ pyramids, (B \& E) spheres, and (C \& F) plates.

Figure 2. (A) Normalized PL and (B) FTIR spectra for ZnO-NPs.

Figure 3. Growth curves for E. coli, K. pneumonia, S. aureus, and S. epidermidis in the presence of increasing concentration of ZnO-NPs synthesized as pyramids, spheres, and plates.

Figure 4. Fraction of cells that partition to the aqueous-hexadecane interface at mid-log versus stationary phase for each organism.

Figure 5. Comparison of the dose response on the growth rate of S. epidermidis by $\mathrm{ZnO}$ pyramids, spheres, and plates for units of (A) mass concentration, (B) surface area, and (C) particle number concentration. Date represent mean $+/-$ standard error. Insets in $\mathrm{B} \& \mathrm{C}$ are expanded view of the data at the lower end of the $\mathrm{x}$-axis to delineate differences in the plates and pyramids.

Figure 6. $\mathrm{ZnO}$ leaching measured by absorbance at $350 \mathrm{~nm}$. 
Figure 7. Box-plots of colony forming units (CFU) per square centimeter of E. coli, S. aureus, and S. epidermidis recovered from bare pegs or pegs coated in $\mathrm{ZnO}$ plates, pyramids, or spheres. Limits of detection for this assay were $100 \mathrm{CFU} / \mathrm{cm}^{2}$

Figure 8. SEM micrographs of (A-C) bare polystyrene pegs, pegs coated in $\mathrm{ZnO}$ (D-F) spheres, (G-I) plates, and (J-L) pyramids cultured with (A, D, G, \& J) E. coli, (B, E, H, \& K) S. aureus, and (C, F, I, \& L) S. epidermidis. Scale bars at the bottom of each image are equal to $10 \mu \mathrm{m}$. 
Table 1. ZnO-NP concentrations for different dosing units

\begin{tabular}{|c|c|c|c|c|c|c|}
\hline $\begin{array}{c}\text { mass } \\
\boldsymbol{\mu g} / \\
\mathbf{m L}\end{array}$ & \multicolumn{2}{|c|}{ [surface area] $\mathbf{~ m}^{2} / \mathbf{L}$} & \multicolumn{4}{|c|}{ [particle \#] } \\
$\mathrm{sp}^{*}$ & $\mathrm{pl}^{\dagger}$ & $\mathrm{py}^{\ddagger}$ & $\mathrm{sp}$ & $\mathrm{pl}$ & $\mathrm{py}$ \\
\hline 167 & 44.7 & 1.36 & 0.69 & 1478 & 45 & 23 \\
333 & 89.2 & 2.72 & 1.38 & 2948 & 90 & 46 \\
500 & 134 & 4.08 & 2.07 & 4426 & 135 & 69 \\
667 & 179 & 5.45 & 2.77 & 5904 & 180 & 91 \\
\hline
\end{tabular}

* spheres, ${ }^{\dagger}$ plates, ${ }^{\ddagger}$ pyramids 

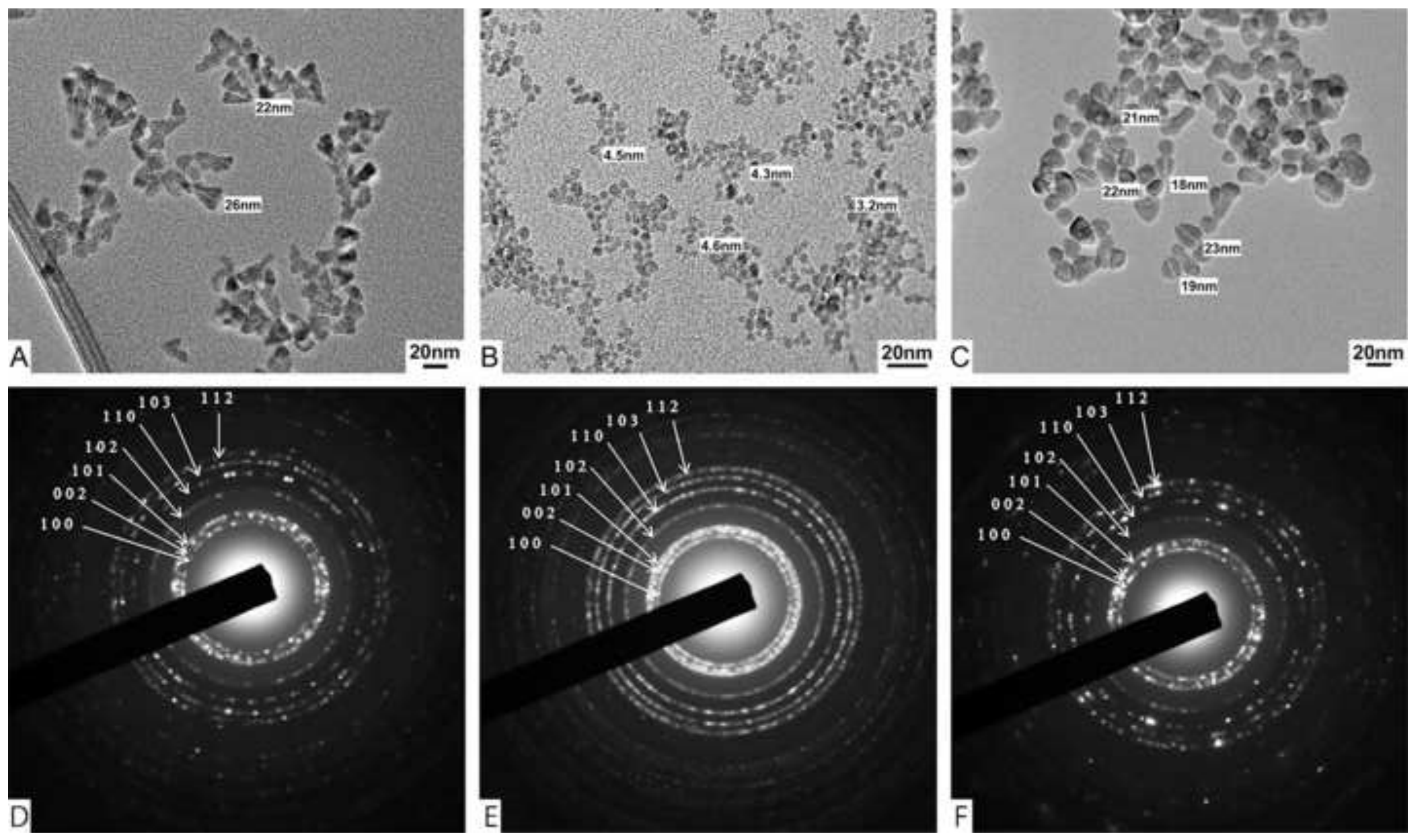

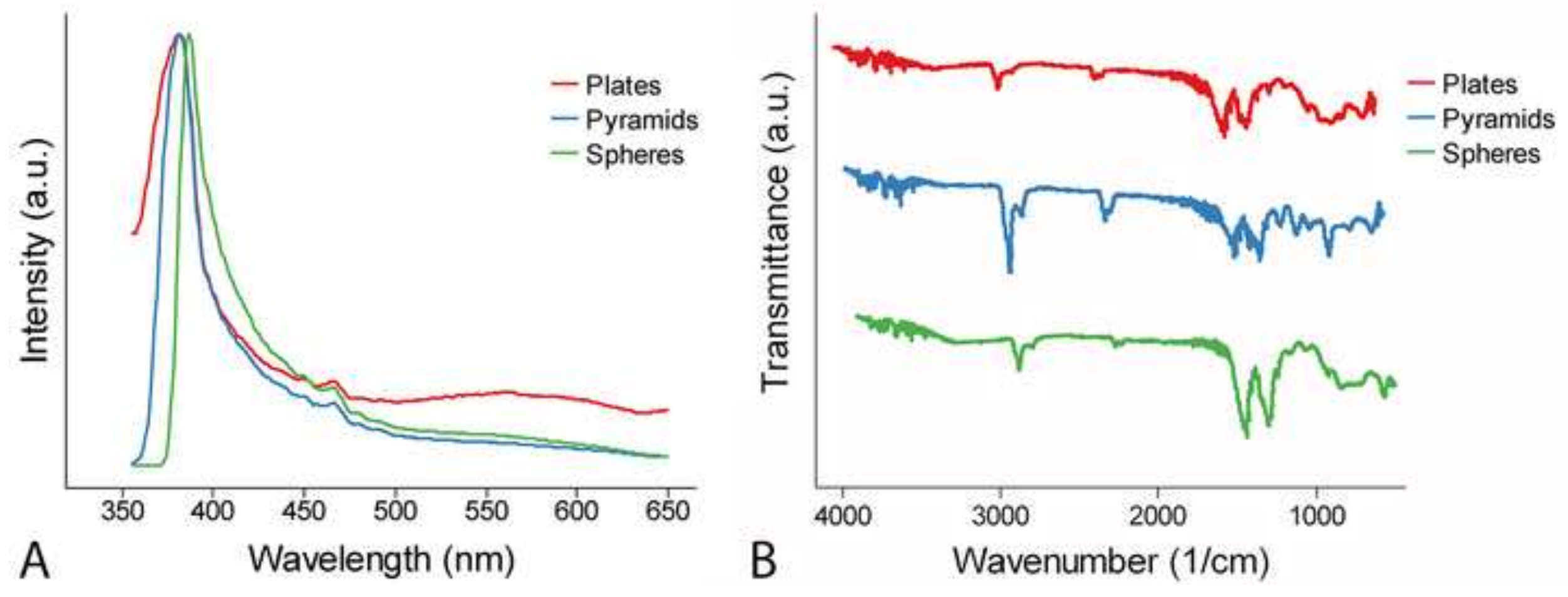


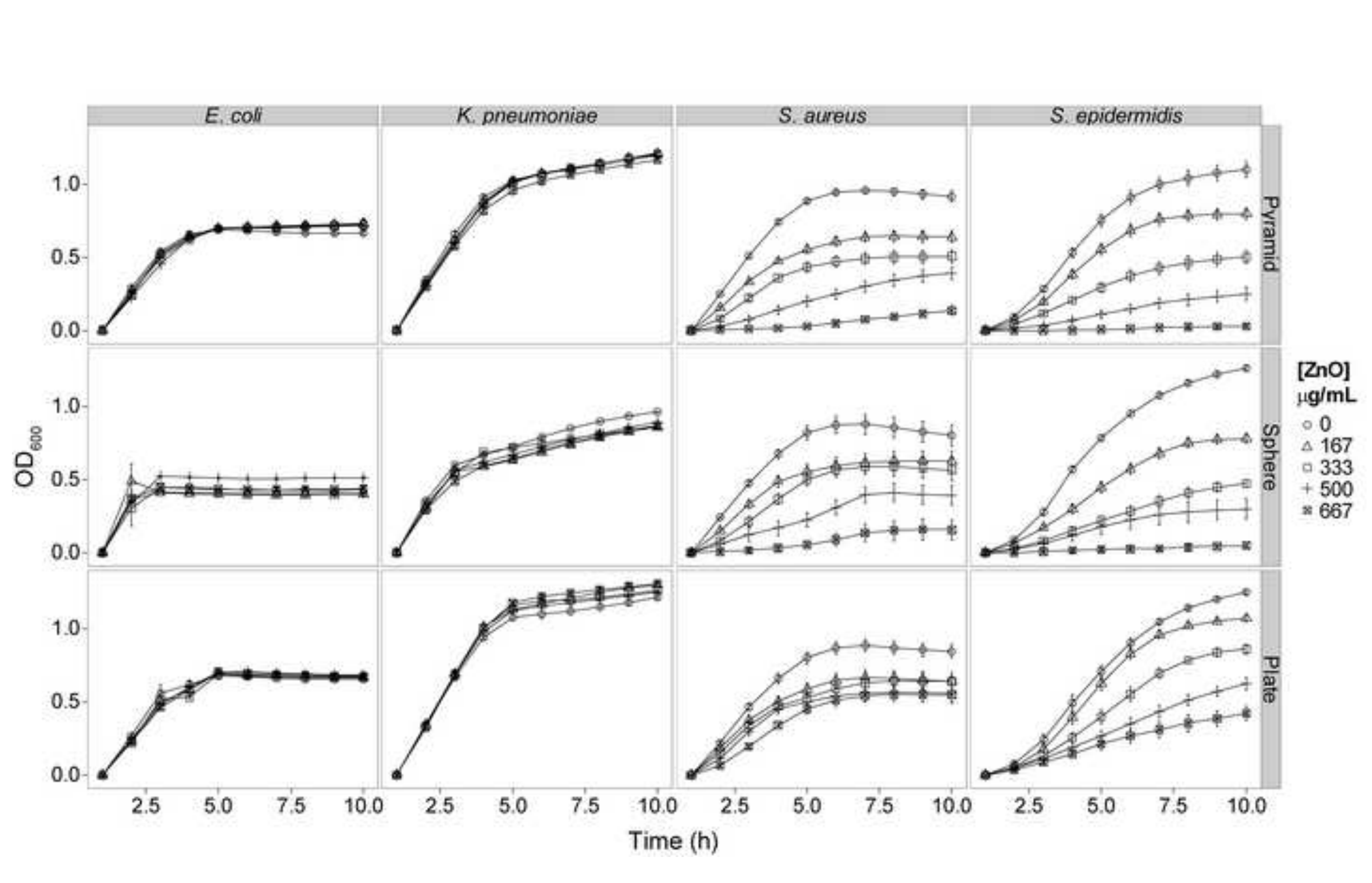

$\mathrm{g} / \mathrm{mL}$

167

500
667 


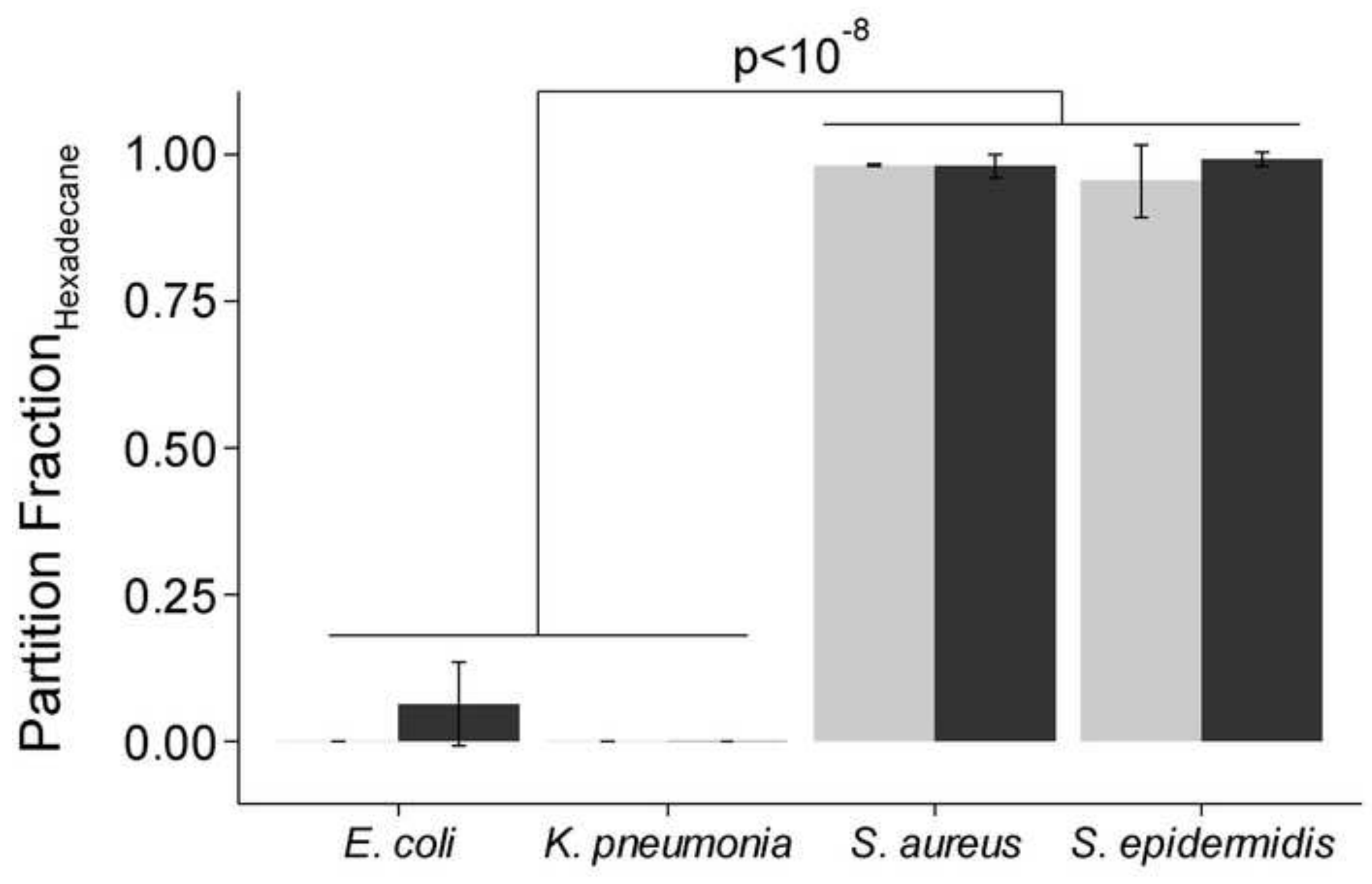

Midlog

Stationary 

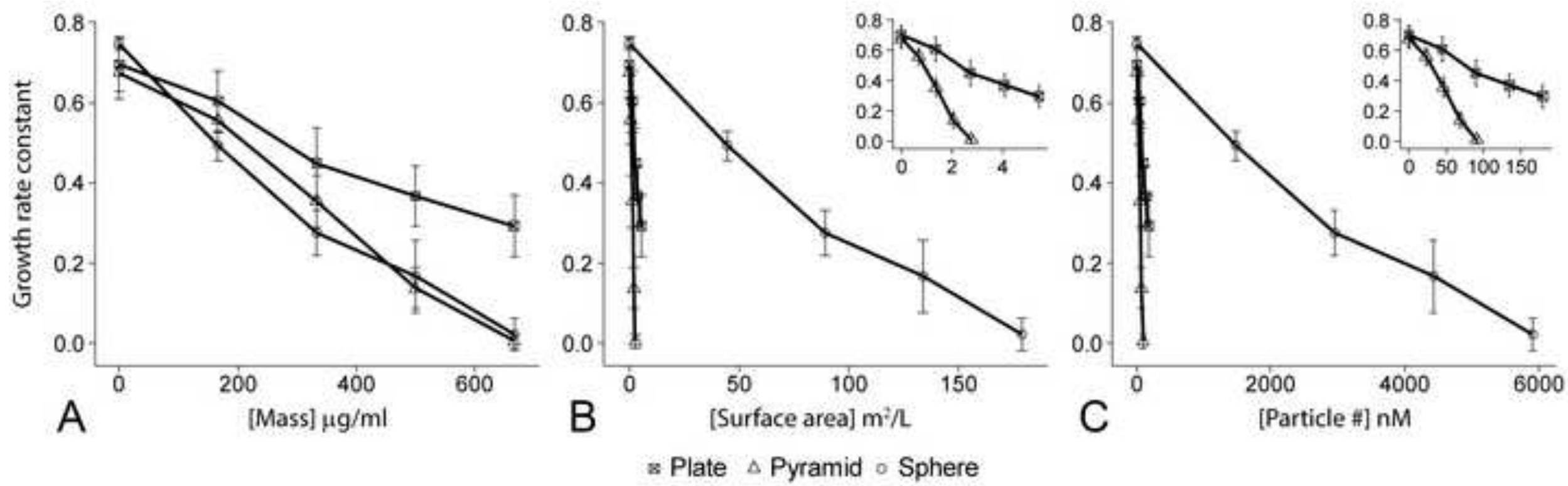


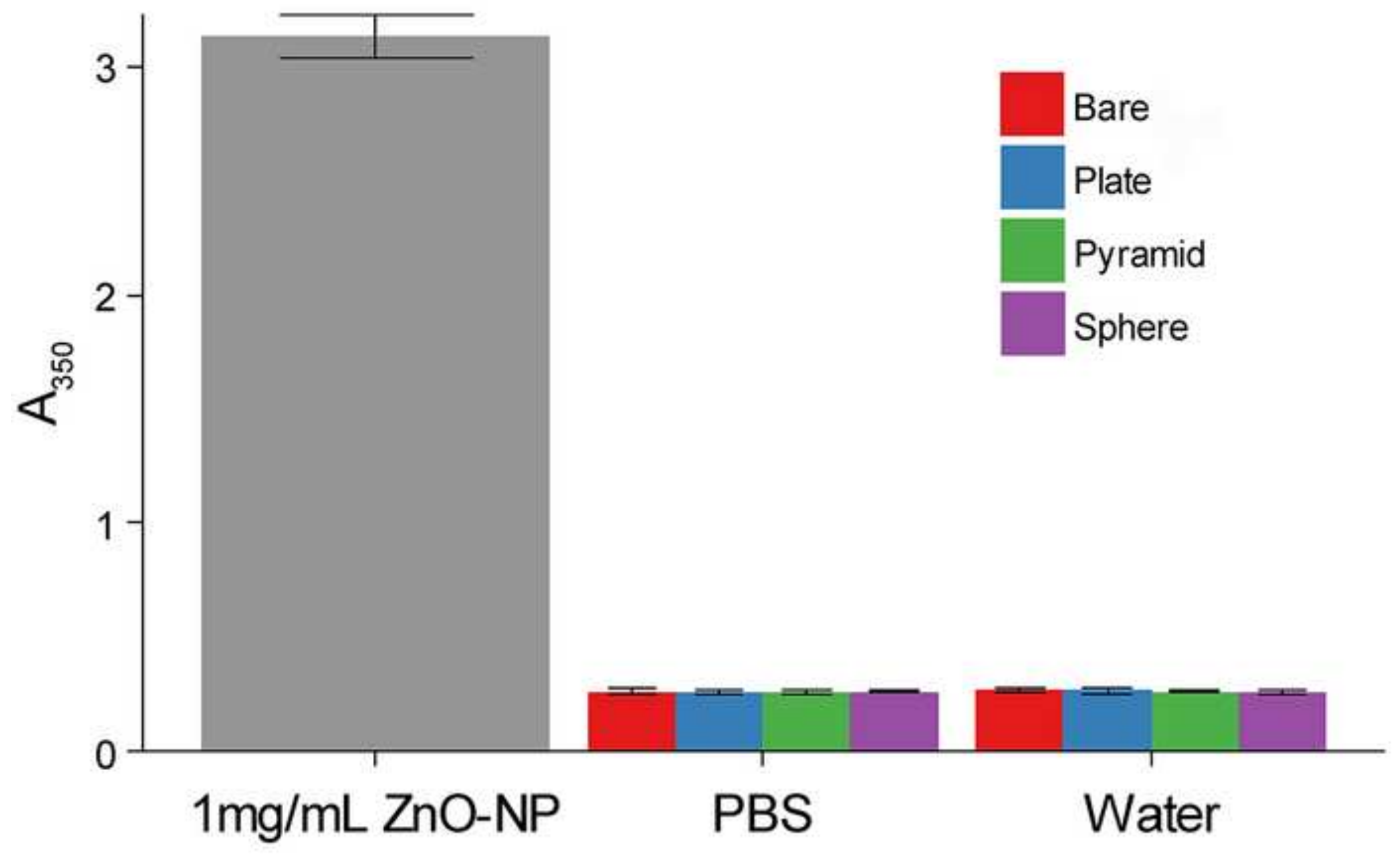




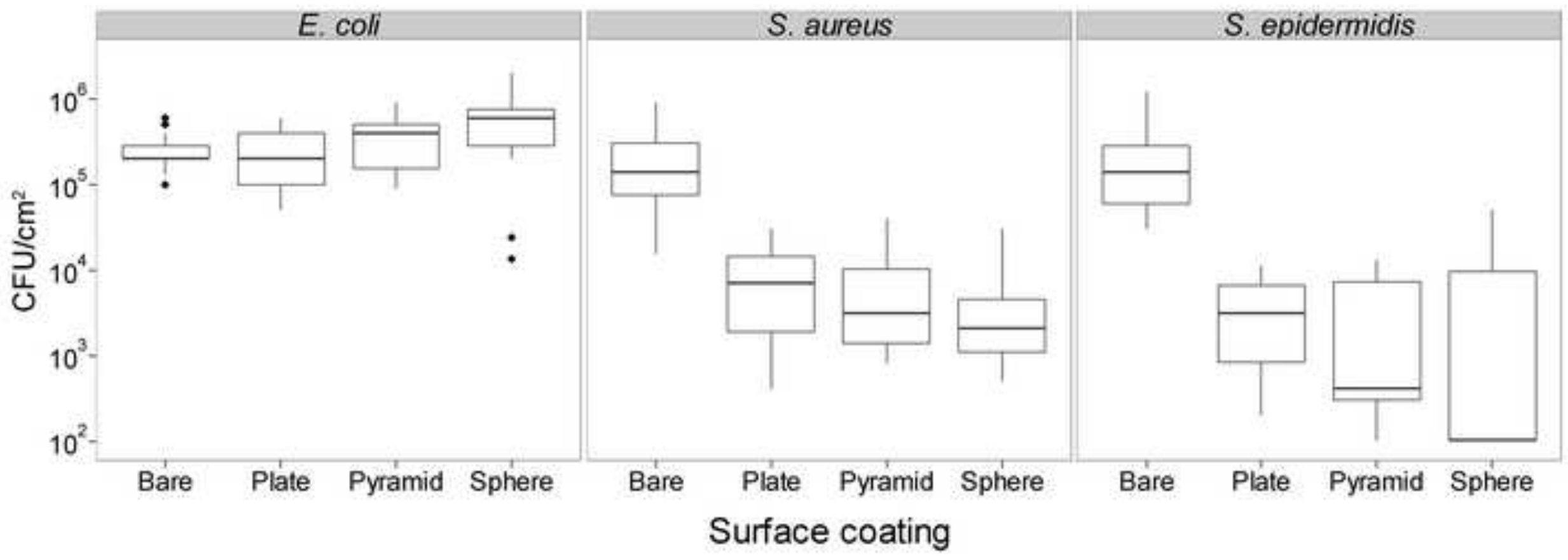




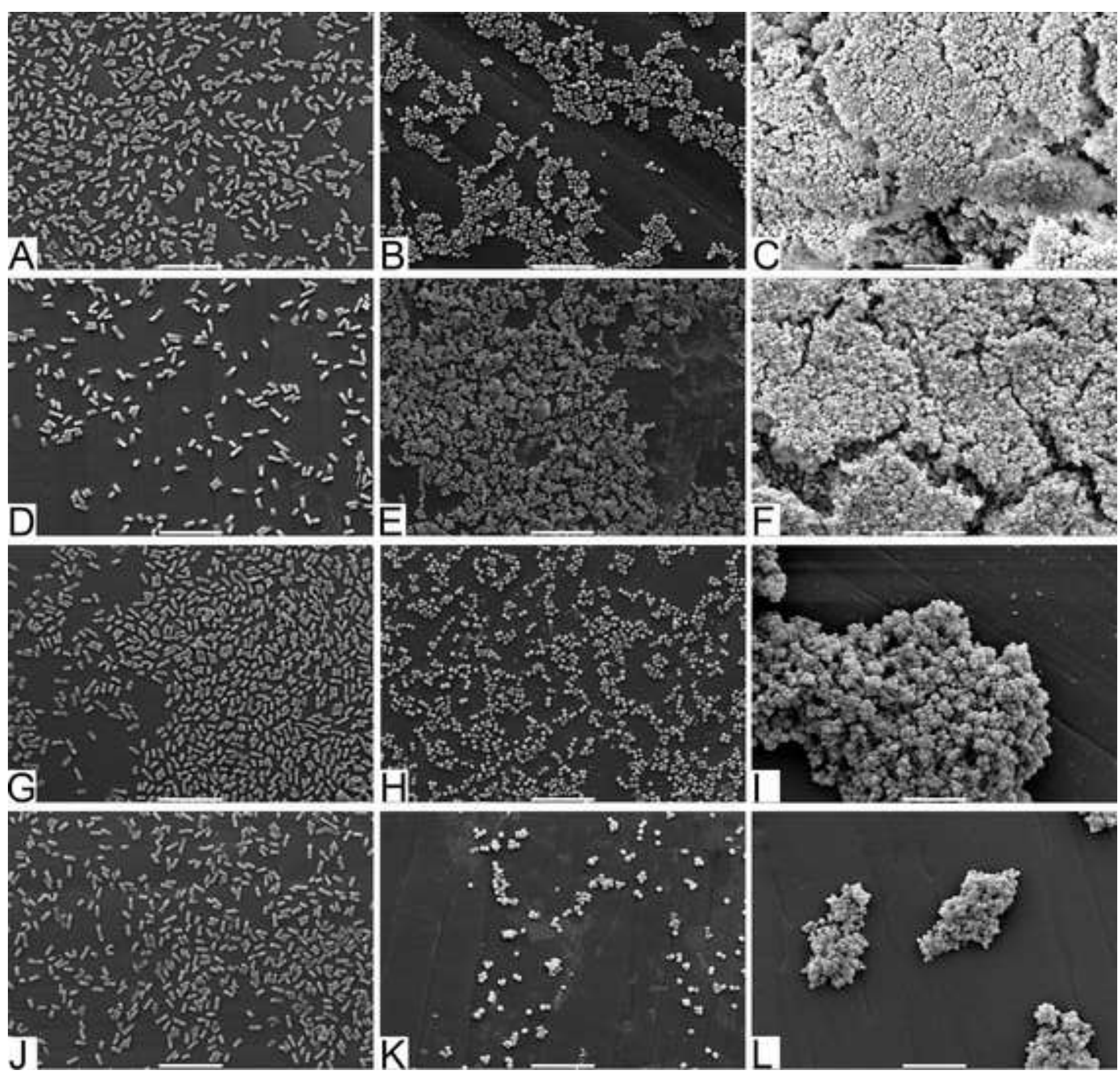


Graphical Abstract

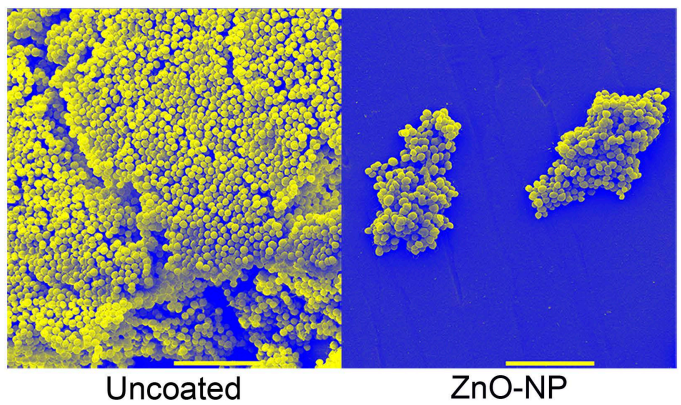

